The Pediatric Infectious Disease Journal Publish Ahead of Print

\title{
DOI: 10.1097/INF.0b013e3182694126
}

Splenomegaly and Variceal Bleeding in a 10-year-old HIV-Infected Girl with Noncirrhotic Portal Hypertension

Vania Giacomet, MD, * Alessandra Viganò, MD,* Francesca Penagini, MD,* Valeria

Manfredini, MD, * Giovanni Maconi, MD, ${ }^{\mathbb{I}}$ Mario Camozzi, MD, and Gian Vincenzo

Zuccotti, MD*

* Department of Pediatrics, L. Sacco Hospital, University of Milan, Italy.

IIDepartment of Gastroenterology, L. Sacco Hospital, University of Milan, Italy.

' Department of Pathology, Niguarda Cà Granda Hospital, Milan, Italy

Abbreviated Title: Noncirrhotic Portal Hypertension in an HIV-Infected Girl

Running Head: Portal Hypertension

The authors have no conflicts of interest or funding to disclose.

\section{Correspondence:}

Dr. Alessandra Viganò.

Department of Pediatrics, L. Sacco Hospital, University of Milan, Italy.

alessandra.vigano@unimi.it 
Abstract: Noncirrhotic portal hypertension (NCPH) is an uncommon liver disease of unknown origin, increasingly described in HIV-infected adults. Prolonged antiretroviral exposure, in particular to didanosine, and thrombophilic predisposition have been suggested as potential pathogenic factors. Data are scant in children. We describe a 10-year-old HIV-infected girl with NCPH who presented with progressive spleen enlargement and variceal bleeding. 
Mortality and morbidity from AIDS-defining disease have decreased dramatically since the introduction of highly active anti-retroviral therapy (HAART) in HIVinfected adults and children ${ }^{1,2}$. As HIV-infected patients live longer, serious non AIDS-defining disease become increasingly important. Novel clinical manifestations or entities may emerge. A series of HIV-infected adults with symptomatic noncirrhotic portal hypertension $(\mathrm{NCPH})$ has recently been described and half of those who underwent histological evaluation showed features compatible with nodular regenerative hyperplasia (NRH) and hepatoportal sclerosis (HPS ${ }^{3-7}$. The following is the description of an HIV-infected girl with a history of splenomegaly who developed NCPH and variceal bleeding.

The patient is a 10-year-old white girl diagnosed with vertically acquired HIV infection at 19 months of age after both parents were diagnosed with HIV infection. HAART was commenced at 2 years of age with zidovudine, nevirapine and lamivudine because of decreased CD4+ count $\left(331 \mathrm{cells} / \mathrm{mm}^{3}\right)$. After 9 months, the antiretroviral regimen was modified to didanosine, tenofovir disoproxil fumarate and lopinavir/ritonavir, due to virological failure. She achieved good virological and immunological control while on this regimen and her growth and development were normal. She was known to have thalassemic trait and iron-deficiency anemia, for which she received oral iron supplementation. At age 10 years, the patient developed progressive spleen enlargement; in June 2011 her spleen was palpable at $10 \mathrm{~cm}$ below the left costal margin, and her liver was not enlarged. At the time of splenomegaly diagnosis, the cumulative exposure to the current antiretroviral treatment was 7 years, HIV-RNA was undetectable ( $<37$ copies/mL) and CD4+ count was 425 cells $/ \mathrm{mm}^{3}$.

The child did not have jaundice, encephalopathy or other stigmata of liver disease. She had no known exposure to toxins or drugs other than mentioned.

Laboratory investigations documented a white blood cell count of $5380 / \mathrm{mm}^{3}$, hemoglobin of $8.5 \mathrm{~g} / \mathrm{dl}$, platelet count of $154000 / \mathrm{mm}^{3}$, international normalized ratio of 1.28 , total bilirubin of $0.79 \mathrm{mg} / \mathrm{dL}$, direct bilirubin of $0.29 \mathrm{mg} / \mathrm{dL}$, albumin of $3.5 \mathrm{~g} / \mathrm{dL}$, serum alanine aminotransferase of $48 \mathrm{IU} / \mathrm{L}$, serum aspartate aminotransferase of $52 \mathrm{IU} / \mathrm{L}, \gamma$-glutamyl transferase of $35 \mathrm{IU} / \mathrm{L}$, alkaline phospatase of $231 \mathrm{IU} / \mathrm{L}$. Serum blood urea nitrogen and creatinine were normal. D-dimers were moderately elevated at $577 \mathrm{ng} / \mathrm{dL}$ (normal value $\leq 250 \mathrm{ng} / \mathrm{dL}$ ). Hypercoagulability studies demonstrated mild decreases in protein $\mathrm{C}$ (antigen and function both $49 \%$ of normal) and free protein $\mathrm{S}$ antigen (60\% of normal). No significant abnormalities of 
homocysteine, activated protein $\mathrm{C}$ resistance, antithrombin III or antibodies to cardiolipin were detected. Genetic analysis did not reveal Factor V Leiden, 5, 10 methylenetetraydrofolate reductase, or prothrombin genetic mutations predisposing to thrombosis.

Moreover, hepatitis $\mathrm{B}$ or $\mathrm{C}$ virus infections were ruled out on the basis of negative serological and molecular tests (COBAS Ampli Prep, Roche Diagnostics, Indianapolis, IN; Branched DNA, Siemens Medical Solutions, Berkley, CA, USA). Circulating autoantibodies (anti-mitochondrial antibodies [AMA], anti-nuclear antibodies [ANA], anti-smooth muscle antibodies [ASMA], anti-liver kidney microsomes antibodies [LKM]) were not detected. Levels of $\alpha-1$ antitrypsin, $\alpha-$ fetoprotein and ceruloplasmin were normal.

Abdominal ultrasonography showed diffuse dishomogeneous hepatic parenchyma, coarse and nodular pattern at segment IV and mild parietal fibrosis of intrahepatic portal veins. Splenomegaly ( $>20 \mathrm{~cm}$ span) and mild ascites $(15 \mathrm{~mL})$ were also noted. Color Doppler demonstrated hepatofugal flow in the portal and splenic venous system as well as suprahepatic vein patency and paraesophageal collateral circulation.

Magnetic resonance imaging revealed a heterogeneous liver with multiple sclerotic nodules. Paraesophageal and perisplenic collaterals as well as ascites were also noted.

Esophagogastroduodenoscopy (EGDS) documented multiple -grade 2 esophageal varices without red wale marks and portal gastropathy without active bleeding. Transjugular suprahepatic catheterization revealed a normal hepatic vein pressure gradient (HVPG: $3.8 \mathrm{mmHg}$, normal $<4 \mathrm{mmHg}$ ) and allowed liver biopsy to be performed.

Histological evaluation showed complete absence of normal portal vein branches in portal tracts which were replaced by small slit-like vessels (Figure 1a), mild to moderate macrovacuolar steatosis, focal sinusoidal fibrosis (Figure 1b) and central mild to moderate venulosclerosis (Figure 1c).

On the whole, ultrasound images, EGDS findings and histological features were consistent with noncirrhotic portal hypertension and hepatoportal sclerosis. After extensive evaluation of other causes, the role of antiretroviral toxicity (particularly didanosine exposure) was hypothesized as a possible contributor to this condition. Therefore, the HAART regimen was changed to darunavir/ritonavir and etravirine. During the four months after change in HAART regimen, the patient was clinically 
stable, maintained undetectable HIV-RNA and CD4+ cell count ranging from 364 to $471 \mathrm{cell} / \mathrm{mm}^{3}$. The scheduled clinical follow-up included assessment of liver function and hepatic ultrasound imaging every three months, and EGDS every six months.

Three months later, liver function tests and abdominal ultrasonography did not show disease progression. At endoscopy, 6 months later, red wale marks were observed on variceal walls. After one month the patient presented hematemesis and was taken to the emergency department. Esophagogastroduodenoscopy revealed multiplegrade 3 esophageal varices; band ligation was performed, and propanolol was initiated. Since discharge she has been well and has not had hematemesis or melena. Her scheduled follow-up includes subsequent endoscopies for variceal monitoring and further banding, regular evaluation of liver function for any elevation of transaminases or reduced hepatic function and liver imaging for monitoring of portal hypertension and ascites. In the case of repeated serious complications of portal hypertension (i.e serious variceal bleeding), transjugular intrahepatic portosystemic shunt (TIPS) would be a feasible option for the patient.

\section{DISCUSSION}

NCPH in HIV-infected adult population has attracted much attention over the last 5 years, following the original description by Maida et al. of 9 subjects with severe liver disease of unknown origin presenting with portal hypertension complicated by variceal bleeding, ascites and/or hepatic encephalopathy ${ }^{8}$. Over the last four years around 90 additional HIV-infected adult patients with NCPH have been reported $5,6,9$. In the majority of these patients liver function tests were generally well preserved with normal albumin, bilirubin, prothrombin levels and mildly elevated serum aminotransferases and alkaline phosphatase. Pathological findings included the absence of advanced liver fibrosis and presence of narrowed and/or obliterated portal veins (hepatoportal sclerosis, HPS) and multiple small nodules (nodular regenerative hyperplasia, NRH). Prolonged prior exposure to didanosine was uniformly recognized. Recently Kochin et al. described a case of a 15-year-old HIVinfected boy with prolonged exposure to didanosine who presented with variceal bleeding and was found to have HPS and NRH on liver biopsy ${ }^{10}$.

Here we describe the second case of NCPH in HIV-infected pediatric population. Our patient like the first pediatric case and the HIV-infected adult cases of NCPH, had prolonged exposure to didanosine. Additionally, she had, as shown in some adult $\mathrm{HIV}$-infected cases of $\mathrm{NCPH}$, mild protein $\mathrm{S}$ and $\mathrm{C}$ deficiency. This finding 
supports the proposed hypothesis of a pathological role of prothrombotic state in development of NCPH. NCPH should be considered an emerging non-AIDS comorbidity in pediatric HIV infection that is likely related to treatment, particularly with didanosine, and other factors.

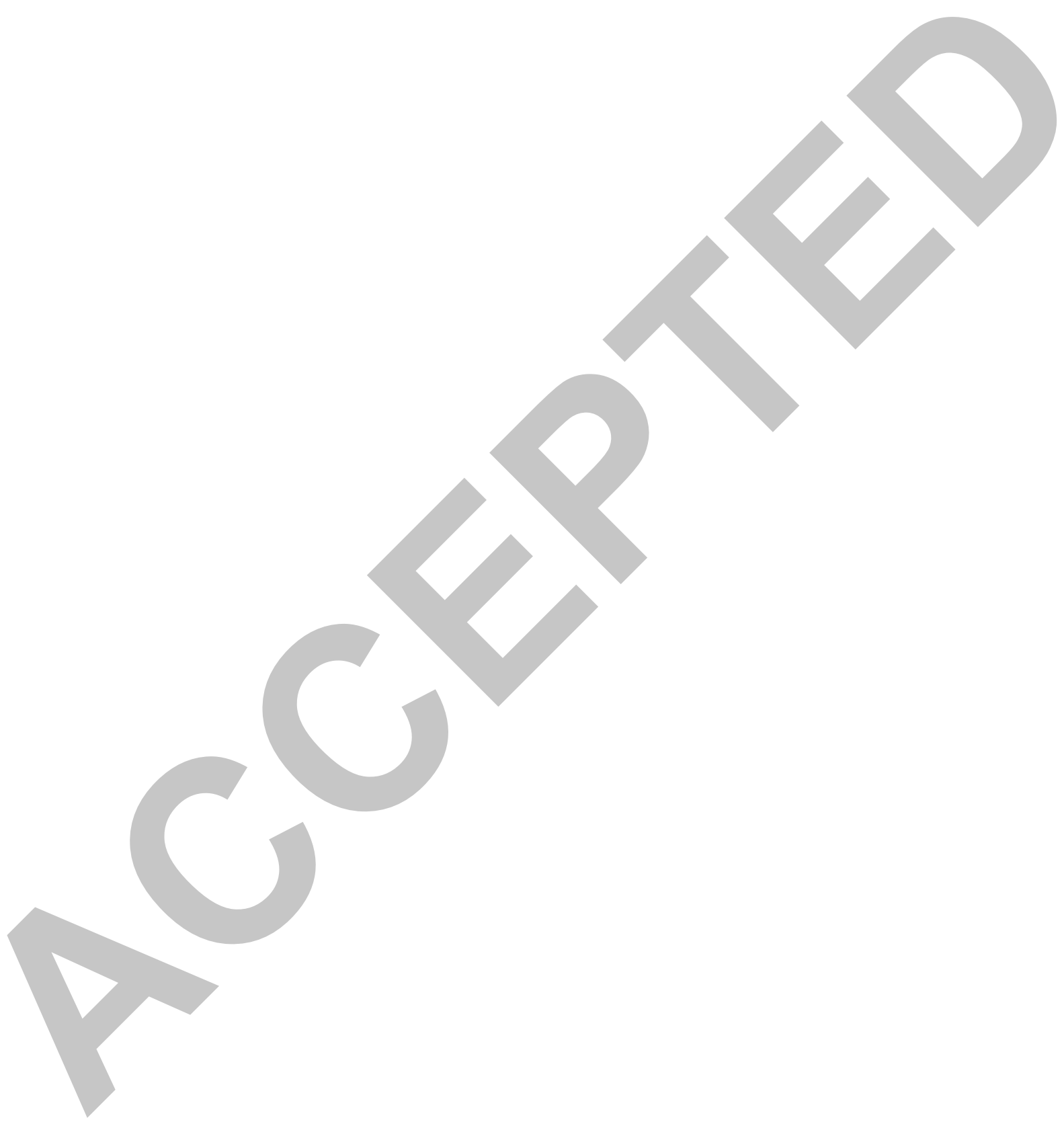




\section{REFERENCES}

1. Antiretroviral Therapy Cohort Collaboration. Life expectancy of individuals on combination antiretroviral therapy in high-income countries: a collaborative analysis of 14 cohort studies. Lancet 2008; 372: 293-9.

2. Brady MT, Oleske JM, Williams PL, et al. Declines in mortality rates and changes of death in HIV-1 infected children during the HAART era. $J$ Acquir Immune Defic Syndr 2010; 53 (1): 86-94.

3. Mallet V, Blanchard P, Verkarre V, et al. Nodular regenerative hyperplasia is a new cause of chronic liver disease in HIV-infected patients. AIDS 2007; 21: 187-92.

4. Sandrine PF, Sylvie A, Andre E, Abdoulaye D, Bernard L, Andre C. Nodular regenerative hyperplasia: a new serious antiretroviral drugs side effect? AIDS 2007; 21: 1498-9.

5. Maida I, Garcia-Gasco P, Sotgiu G, et al. Antiretroviral-associated portal hypertension: a new clinical condition? Prevalence, predictors and outcome. Antivir Ther 2008; 13: 103-7.

6. Cesari M, Schiavini M, Marchetti G, et al. Noncirrhotic Portal Hypertension in HIV-Infected Patients: A case Control Evaluation and Review of the Literature. AIDS Patient Care and STDs 2010; 24 (11): 697-703.

7. Vispo E, Moreno A, Maida I, et al. Noncirrhotic portal hypertension in HIVinfected patients: unique clinical and pathological findings. AIDS 2010, 24: 1171-1176.

8. Maida I, Nunez M, Rios MJ, et al. Severe liver disease associated with prolonged exposure to antiretroviral drugs. J Acquir Immune Defic Syndr 2006; 42:177-182

9. Vispo E, Morello J, Rodriguez-Novoa S, et al. Noncirrhotic portal hypertension in HIV infection. Curr Opin Infect Dis 2011 24: 12-18.

10. Kochin I, Margret M, Arnon R, et al. Variceal Bleeding in an Adolescent With HIV Diagnosed With Hepatoportal Sclerosis and Nodular Regenerative Hyperplasia. J Pediatr Gastroenterol Nutr 2010, 50 (3): 340-343. 


\section{Figure Legends}

Fig 1. A. Portal tract. Slit-like portal vein branch (arrow). H\&E stain. Fig 1. B. Central vein fibrosis and sinusoidal fibrosis. Sirius-Red stain. Fig 1. C. Central vein. Moderate degree of fibrosis (arrows). H\&E stain.

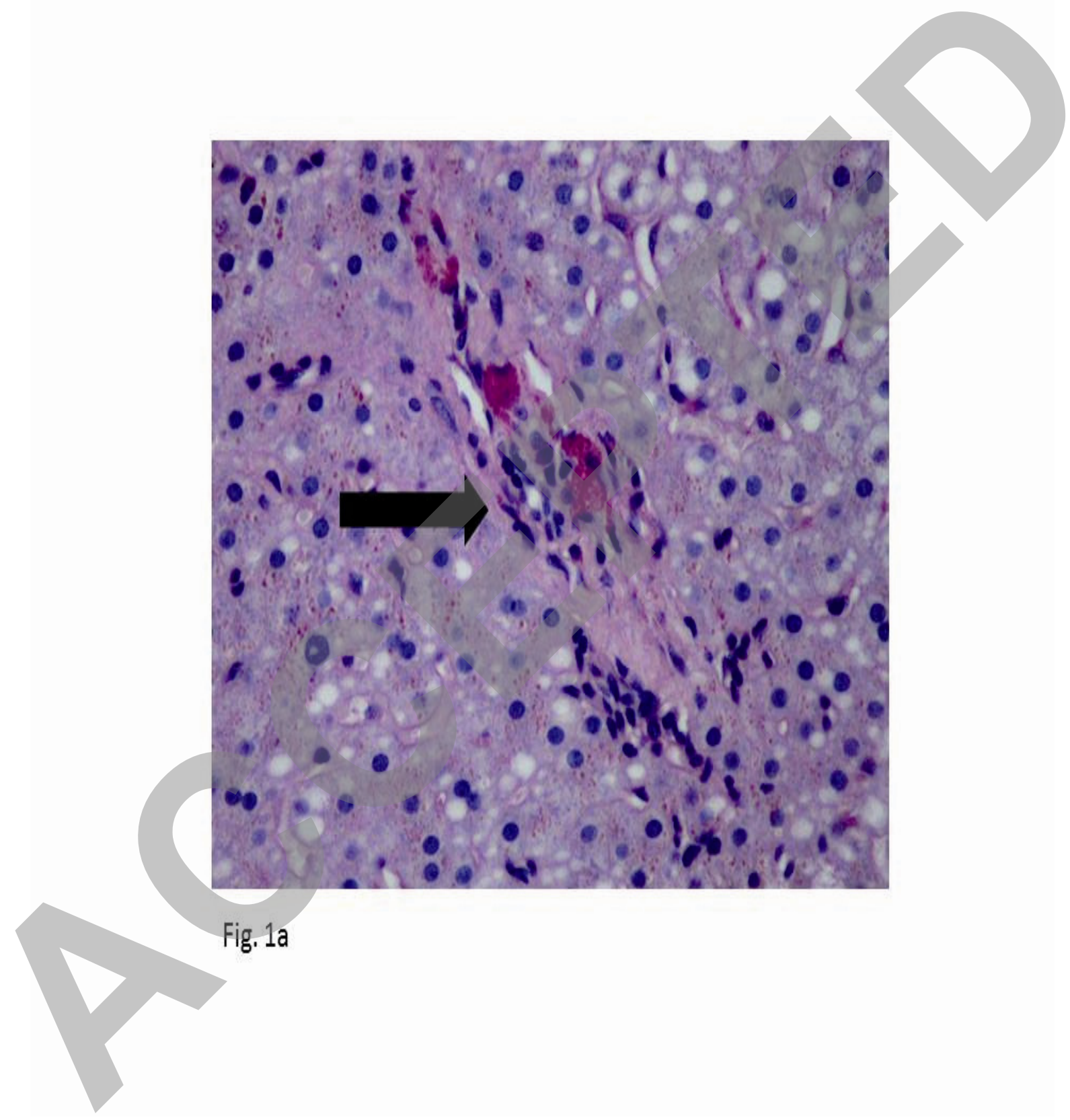




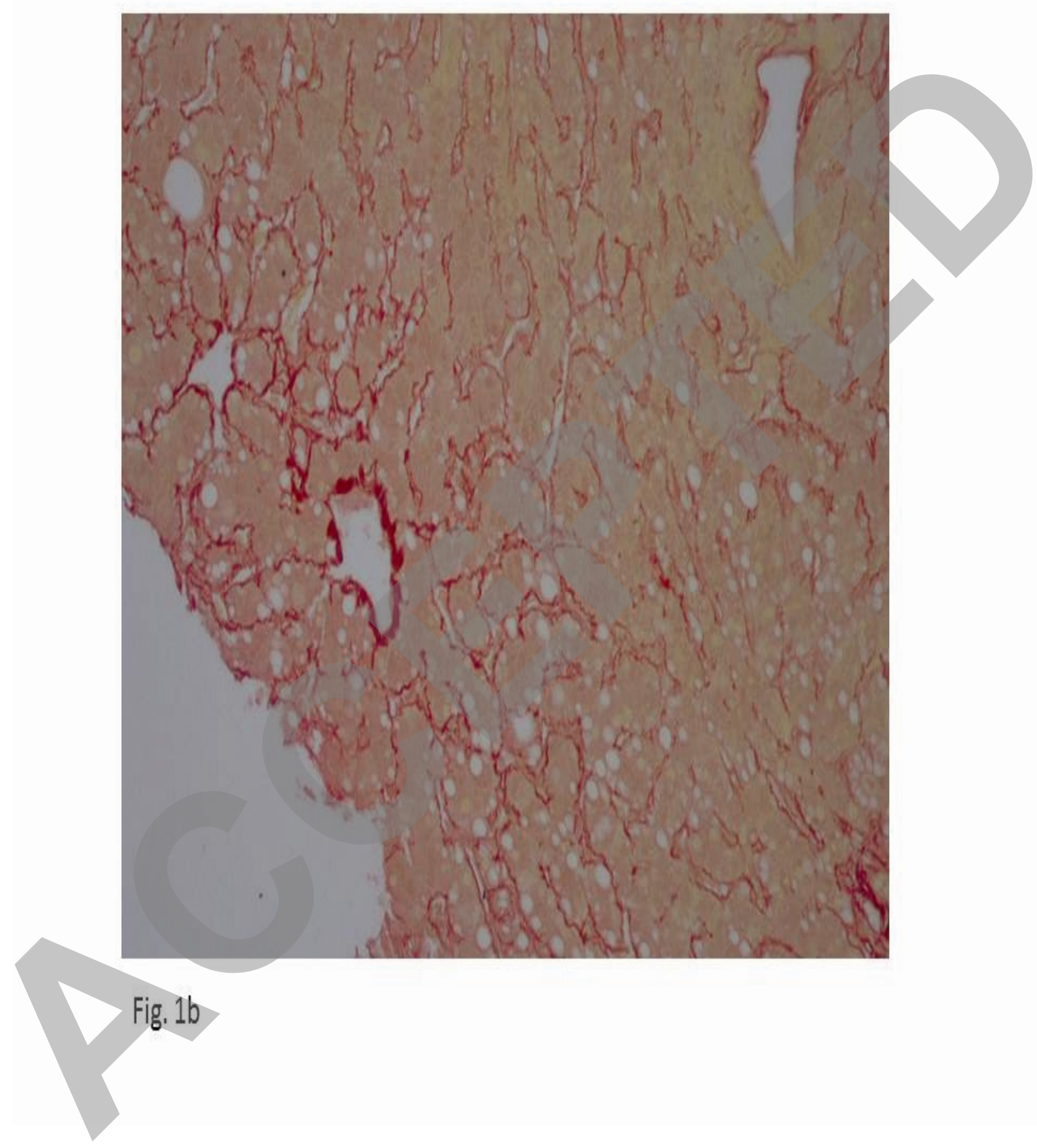

Copyright @ 2012 Lippincott Williams \& Wilkins. Unauthorized reproduction of this article is prohibited. 


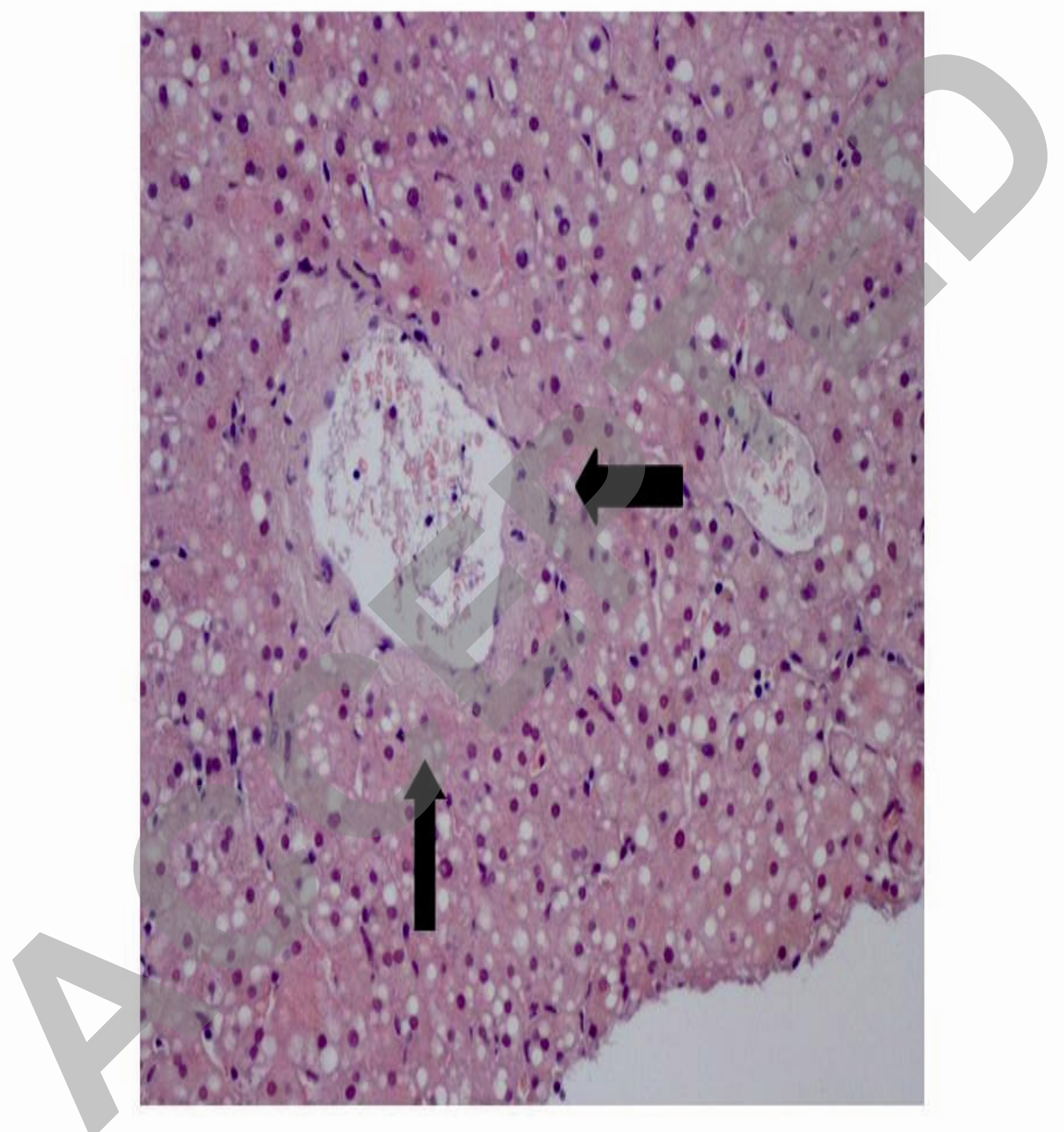

Fig. 10

Copyright @ 2012 Lippincott Williams \& Wilkins. Unauthorized reproduction of this article is prohibited. 